\section{Morphometric studies on the passive role of menisci in upward fixation of the patella in buffalo and cattle}

\author{
Abdalla Hifny, \\ Kamal Eldin Hashem Abdalla, \\ Yousria A. Abdel Rahman, Khaled Aly, \\ Ruwaida Abdelmoaty Elhanbaly \\ Department of Anatomy and Histology, \\ Faculty of Veterinary Medicine, Assiut \\ University, Assiut, Egypt
}

\section{Abstract}

The present study showed that the medial and lateral compartments of the femorotibial joint have an intervening meniscus located between the femur and tibia. The lateral meniscus is semicircular and covers a larger portion of the tibial condyle than the medial meniscus. The lateral meniscus has cranial and caudal meniscal ligaments anchoring it to the tibia. The present investigation shows that the dimensions of the meniscal ligaments of buffalo and cattle are almost the same except for the caudal ligament of lateral meniscus and the meniscofemoral ligament. The caudal ligament of lateral meniscus is longer in buffalo than in cattle. Therefore, the femur moves more cranially than normal to straighten the joint. The meniscofemoral ligament is thicker in cattle $(6.55 \pm 0.04 \mathrm{~mm})$ than in buffalo $(3.85 \pm 0.05 \mathrm{~mm})$. Therefore, it helps to stabilize the movement of the menisci. Furthermore, the presence of the transverse genual ligament which fixes the two menisci cranially increases stability and prevents a more forward movement of the femur.

\section{Introduction}

In Egypt, buffalo and cattle are considered the main source of meat production.

The femorotibial joint is the largest joint in the body and is comprised of two condyloid articulations. The medial and lateral femoral condyles articulate with the corresponding tibial plateau. Intervening medial and lateral menisci serve to enhance the conformity of the joint. ${ }^{1}$ The meniscus and cruciate ligaments play an important role in weight bearing and stability of the stifle joint.

The available literature shows that the incidence of upward fixation of the patella is higher in buffalo than in cattle. ${ }^{2}$ This fact may refer to anatomical structures of this joint in these animals. This study aims to examine the anatomical features and morphometry of the menisci in buffalo and cattle to indicate their passive roles in inducing upward fixation of the patella in both these animals.

\section{Materials and Methods}

This study was carried out on 10 fresh specimens of stifle regions in buffalo and cattle, 5 for each adult animal of local breeds (age 2-2.5 years). Buffalo weighed approximately 500-550 $\mathrm{kg}$ and cattle weighed approximately 450-600 $\mathrm{kg}$. Specimens were collected from Assiut (Egypt) slaughter house. The studied specimens were clinically healthy and showed no evidence of marked bony abnormalities or degenerative diseases.

Anatomical study of the mensci included: i) morphological study: study of the morphological features of the menisci of the stifle joint depends mainly on demonstration of the shape and extension of its articular surfaces as well as the shape, density, extension and area of attachment of the meniscal ligaments; ii) morphometrical study: different measurements including the length, width and thickness of the menisci as well as the length, width and thickness of the ligaments. All the anatomical components were photographed and the dimensions were recorded using a digital caliper.

All measurements were statistically analyzed using a one way ANOVA. Descriptive statistics are given as means \pm SD (standard deviation). The statistical analysis was carried out using Microsoft ${ }^{\circledR}$ Excel 2007. Data are presented in tables. The nomenclature used in the present work was adapted to the Nomina Anatomica Veterinaria as well as the available literature. ${ }^{3}$

\section{Results}

The articular surfaces of the femorotibial joint include the condyles of the femur, the condyles of the tibia and the intervening medial and lateral meniscus.

Results of our comparative study include relative but not absolute measurements.

\section{Medial meniscus}

The medial meniscus (Table 1, Figures 1-4) is a fibrocartilaginous plate that partially divides the medial femorotibial sac of the femorotibial joint into proximal and distal compartments. It covers the medial condyle of the tibia except its axial part. It is thin and crescent-shaped. Therefore, it has two surfaces (proximal and distal), two borders (axial
Correspondence: Khaled H. Aly, Department of Anatomy and Histology, Faculty of Veterinary Medicine, Assiut University, Assiut, Egypt.

Tel. +20.1008047754 - Fax: +20.882366503

E-mail: khaledali69@hotmail.com

Key words: menisci, buffalo, cattle.

Conflict of interests: the authors report no conflict of interests.

Received for publication: 21 July 2012.

Revision received: 11 September 2012.

Accepted for publication: 21 September 2012.

This work is licensed under a Creative Commons Attribution NonCommercial 3.0 License (CC BYNC 3.0).

(C) Copyright A. Hifny et al., 2012

Licensee PAGEPress srl, Italy

Veterinary Science Development 2012; 2:e14

doi:10.4081/vsd.2012.e14

and abaxial), and two extremities (cranial and caudal).

The level of its proximal surface is lower than that of the lateral meniscus as the medial condyle of the femur is lower than that of the lateral one. The surface is strongly concave cranoicaudally and transversally. The distal surface is adapted to the medial condyle of the tibia which is nearly flattened.

The axial border of the medial meniscus is thin and concave. It forms a U-shaped notch: $19.54 \pm 0.04 \mathrm{~mm}$ deep in buffalo and $12.56 \pm 0.02$ $\mathrm{mm}$ deep in cattle. The distance between both borders is nearly the same: approximately $23.66 \pm 0.23 \mathrm{~mm}$ in both animals.

The abaxial border of the medial meniscus corresponds to the outer margin of the medial condyle of the tibia. It has no muscular or ligamentous attachments and is related medially to the medial collateral ligaments of the femorotibial joint.

The thickness of this border varies cranially, in the middle and caudally: $14.71 \pm 0.24$, $11.19 \pm 0.09$ and $14.75 \pm 0.06 \mathrm{~mm}$, respectively, in buffalo and $13.92 \pm 0.03,8.52 \pm 0.17$ and $12.38 \pm 0.04 \mathrm{~mm}$, respectively, in cattle. As a result, this border is thinner in the middle than at either extremity. Furthermore, both extremities are almost the same thickness.

The cranial extremity is approximately $19.44 \pm 0.16 \mathrm{~mm}$ wide in buffalo and $21.82 \pm 0.07$ $\mathrm{mm}$ in cattle. The cranial ligament of the medial meniscus is attached to this extremity. The caudal extremity is thicker and narrower than the cranial one. It measures $17.45 \pm 0.19 \mathrm{~mm}$ wide in buffalo and $19.75 \pm 0.06 \mathrm{~mm}$ in cattle.

\section{Lateral meniscus}

The lateral meniscus (Table 1, Figures 1-4) is a fibrocartilaginous plate that partially 
divides the lateral sac of the femorotibial joint into proximal and distal compartments. It covers the articular surface of the lateral condyle of the tibia. Its axial part covers towards the lateral tubercle of the intercondyloid eminence and the caudolateral margin where the tendon of origin of the popliteus is located separating it from the lateral collateral ligament of the femorotibial joint.

The lateral meniscus is crescent-shaped and, therefore, has two surfaces (proximal and distal) two borders (axial and abaxial) and two extremities (cranial and caudal).

The proximal surface forms the floor of the proximal compartment of the lateral femorotibial sac. This surface is adapted to the lateral condyle of the femur; therefore, it is strongly concave craniocaudally and transversally. The distal surface forms the roof of the distal compartment of the lateral femorotibial sac. This surface is adapted to the saddleshaped lateral condyle of the tibia. It is less curved than the proximal surface.

The axial border of the lateral meniscus is thin and concave. It forms a U-shaped notch. Therefore, the uncovered part of the lateral condyle of the tibia represents the area of communication between the proximal and distal compartments of the lateral sac of the femorotibial joint. In turn, in this area there is a direct contact between the lateral condyle of the tibia and the lateral condyle of the femur. The notch is $12.59 \pm 0.04 \mathrm{~mm}, 12.46 \pm 0.03 \mathrm{~mm}$ deep while the distance between the two borders is approximately $27.91 \pm 0.03 \mathrm{~mm}$ and $25.84 \pm 0.01 \mathrm{~mm}$ in buffalo and cattle, respectively.

The abaxial border is convex and its thickness varies cranially, in the middle and caudally: approximately19.35 $\pm 0.06,15.55 \pm 0.12$ and $23.93 \pm 0.13 \mathrm{~mm}$, respectively, in buffalo and $16.31 \pm 0.03 \mathrm{~mm}, 12.71 \pm 0.03 \mathrm{~mm}$ and $22.032 \pm 0.02 \mathrm{~mm}$, respectively, in cattle. Therefore, the abaxial border is thickest cranially and thinner in the middle than caudally. A groove has been observed on the lateral aspect of the outer border where the tendon of origin of popliteal muscle operates. The meniscofemoral ligament and the caudal ligament of the lateral meniscus are attached to the caudal part of the abaxial border.

The synovial membrane of the proximal and distal compartments of the lateral sac of the femorotibial joint is attached to the proximal and distal margins of the abaxial border, respectively.

The cranial extremity of the lateral meniscus is narrow: approximately $23.49 \pm 0.6 \mathrm{~mm}$ wide in buffalo and $22.19 \pm 0.02 \mathrm{~mm}$ in cattle. The caudal extremity is thicker and broader than the cranial one: approximately $29.37 \pm 0.08 \mathrm{~mm}$ wide in buffalo and $26.75 \pm 0.06$ $\mathrm{mm}$ in cattle.

The ligaments of the menisci are divided into two groups: ligaments of the medial meniscus and ligaments of the lateral meniscus.

\section{Ligaments of medial meniscus}

\section{Cranial ligament of medial meniscus}

The cranial ligament of the medial meniscus (Table 2, Figures 5-8) is attached to the cranial extremity of the medial meniscus. It extends laterally and slightly distally ending in a shallow depression in the cranial intercondyloid area cranial to the attachment of the cranial part of the cranial cruciate ligament. Length, width and thickness are, respectively, $30.76 \pm 0.4,16.7 \pm 0.23$ and $6.49 \pm 0.2 \mathrm{~mm}$ in buffalo and $25.82 \pm 0.12 \mathrm{~mm}, 20.71 \pm 0.04 \mathrm{~mm}$ and $3.46 \pm 0.01 \mathrm{~mm}$, respectively, in cattle.

\section{Caudal ligament of medial meniscus}

The caudal ligament of the medial meniscus is thicker than the cranial ligament. It arises from the caudal extremity of the medial meniscus. It extends cranially and laterally ending in the deep and large caudal intercondyloid area of the tibia.

Length, width and thickness of the caudal ligament of the medial meniscus are, respectively, $20.29 \pm 0.25,18.26 \pm 0.04$ and $4.32 \pm 0.03$ $\mathrm{mm}$ in buffalo and $25.42 \pm 0.04 \mathrm{~mm}, 13.56 \pm 0.05$ $\mathrm{mm}$ and $4.71 \pm 0.03 \mathrm{~mm}$, respectively, in cattle.

\section{Ligaments of the lateral meniscus}

Cranial ligament of the lateral meniscus

The cranial ligament of the lateral meniscus

Table 1. Dimensions of the articular surfaces of the menisci in buffalo and cattle ( $\mathrm{mm})$.

\begin{tabular}{llr} 
& Buffalo & Cattle \\
Medial meniscus & & \\
Thickness of abaxial border & & \\
Cranially & $14.71 \pm 0.24$ & $13.92 \pm 0.03$ \\
At the middle & $11.19 \pm 0.09$ & $8.52 \pm 0.17$ \\
Caudally & $14.75 \pm 0.06$ & $12.38 \pm 0.04$ \\
Width of cranial extremity & $19.44 \pm 0.16$ & $21.82 \pm 0.07$ \\
Width of caudal extremity & $17.45 \pm 0.19$ & $19.75 \pm 0.06$ \\
Distance between two borders & $23.66 \pm 0.23$ & $23.16 \pm 0.03$ \\
Depth of notch & $19.54 \pm 0.04$ & $12.56 \pm 0.02$ \\
Lateral meniscus & & \\
Thickness of abaxial border & & \\
Cranially & $19.35 \pm 0.06$ & $16.31 \pm 0.03$ \\
At the middle & $15.55 \pm 0.12$ & $12.71 \pm 0.03$ \\
Caudally & $23.93 \pm 0.13$ & $22.32 \pm 0.02$ \\
Width of cranial extremity & $23.49 \pm 0.6$ & $22.19 \pm 0.02$ \\
Width of caudal extremity & $29.37 \pm 0.08$ & $27.85 \pm 0.06$ \\
Distance between two borders & $27.91 \pm 0.03$ & $25.84 \pm 0.01$ \\
Depth of notch & $12.59 \pm 0.04$ & $12.46 \pm 0.02$ \\
\hline
\end{tabular}

Table 2. Dimensions of the meniscal ligaments in buffalo cattle ( $\mathrm{mm})$.

\begin{tabular}{lrr} 
Meniscal ligaments & Buffalo & Cattle \\
Ligaments of medial meniscus & & \\
1. Cranial ligament & & \\
Length & $30.76 \pm 0.40$ & $25.82 \pm 0.12$ \\
Width & $16.7 \pm 0.23$ & $20.71 \pm 0.04$ \\
Thickness & $6.49 \pm 0.20$ & $3.46 \pm 0.01$ \\
2. Caudal ligament & & \\
Length & $20.29 \pm 0.25$ & $25.42 \pm 0.04$ \\
Width & $18.26 \pm 0.04$ & $13.56 \pm 0.05$ \\
Thickness & $4.32 \pm 0.03$ & $4.71 \pm 0.03$ \\
Ligaments of lateral meniscus & & \\
1. Cranial ligament & & \\
Length & $34.34 \pm 0.26$ & $39.57 \pm 0.05$ \\
Width & $19.39 \pm 0.20$ & $13.05 \pm 0.02$ \\
Thickness & $4.11 \pm 0.02$ & $3.55 \pm 0.01$ \\
2. Caudal ligament & & \\
Length & $38.61 \pm 0.14$ & $31.49 \pm 0.19$ \\
Width & $28.57 \pm 0.15$ & $11.82 \pm 0.31$ \\
Thickness & $2.88 \pm 0.05$ & $2.38 \pm 0.28$ \\
3. Meniscofemoral & & \\
Length & $49.62 \pm 0.13$ & $50.92 \pm 0.06$ \\
Width & $14.87 \pm 0.12$ & $14.58 \pm 0.08$ \\
Thickness & $3.85 \pm 0.05$ & $6.55 \pm 0.04$ \\
\hline
\end{tabular}


(Table 2, Figures 5-8) is attached to the cranial extremity and adjacent abaxial border of the lateral meniscus. It extends medially and slightly distally on the lateral tibial condyle to terminate in the cranial intercondyloid area between the areas of attachment of the cranial and caudal parts of the cranial cruciate ligament. Length, width and thickness are $34.34 \pm 0.26,19.39 \pm 0.20$ and $4.11 \pm 0.02 \mathrm{~mm}$, respectively, in buffalo and $39.57 \pm 0.05$, $13.05 \pm 0.02$ and $3.55 \pm 0.01 \mathrm{~mm}$, respectively, in cattle.

\section{Caudal ligament of the lateral meniscus}

The caudal ligament is a thin fibrous sheet. It arises from the caudal part of the thick abaxial border of the lateral meniscus. It extends distally and slightly medially to turn around the caudal margin of the lateral tibial condyle ending just below it in the popliteal notch that undercovers the distal portion of the caudal cruciate ligament. Length, width and thickness are $38.61 \pm 0.14 \mathrm{~mm}, 28.57 \pm 0.15 \mathrm{~mm}$ and
$2.88 \pm 0.05 \mathrm{~mm}$, respectively, in buffalo and $31.49 \pm 0.19 \mathrm{~mm}, 11.82 \pm 0.31 \mathrm{~mm}$ and $2.38 \pm 0.28$ $\mathrm{mm}$, respectively, in cattle.

\section{Meniscofemoral ligament}

The meniscofemoral ligament is a thick well-developed fibrous band. It is attached to the proximocaudal part of the abaxial border of the lateral meniscus. It extends proximally and slightly cranially in the intercondyloid fossa to terminate in the proximomedial part of this fossa close to the medial condyle of the femur. Length, width and thickness are $49.62 \pm 0.13$ $\mathrm{mm}, 14.87 \pm 0.12 \mathrm{~mm}$ and $3.85 \pm 0.05 \mathrm{~mm}$, respectively, in buffalo and $50.92 \pm 0.06 \mathrm{~mm}$, $14.58 \pm 0.08 \mathrm{~mm}$ and $6.55 \pm 0.04 \mathrm{~mm}$, respectively, in cattle.

\section{Transverse genual ligament}

The transverse genual ligament (Figure 8) is a thin transverse band found only in cattle and extends between the cranial ligament of the medial and lateral menisci. It is located between the cranial ligament of the medial meniscus cranially and the cranial part of the cranial cruciate ligament caudally.

\section{Discussion}

The general morphological features of the menisci entering in the formation of the femorotibial joint of buffalo and cattle show great resemblance to those of the $0 x,{ }^{4}$ camel, ${ }^{5}$ horse, ${ }^{6}$ and sheep. ${ }^{7}$

The present study shows that the axial border of the menisci is thin and concave forming a U-shaped notch. The proximal and distal compartments of the medial and lateral sacs of the femorotibial joint are connected through this notch. Also there is a direct contact between the condyles of the tibia and femur. The area of contact is large on the medial side in cattle and on the lateral side in buffalo. This notch is $19.54 \pm 0.04 \mathrm{~mm}$ and $12.56 \pm 0.02 \mathrm{~mm}$
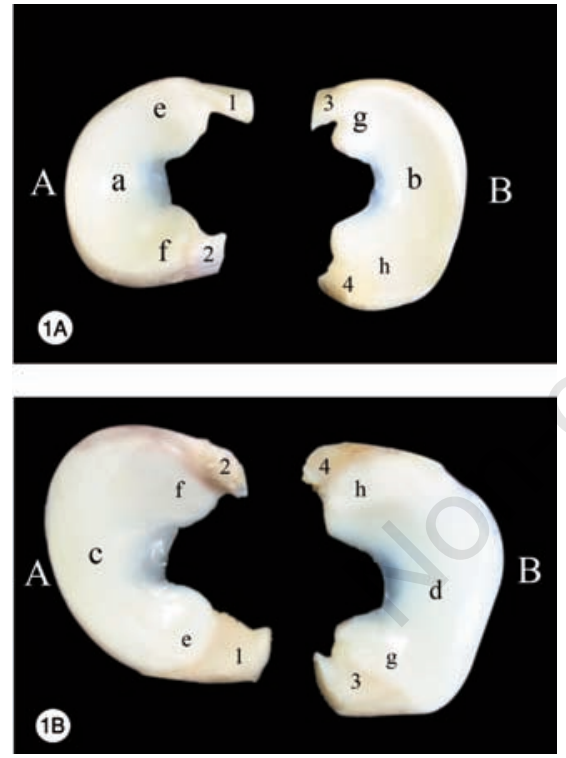

Figure 1. Photograph of menisci of right stifle of buffalo. A) Medial meniscus, B) lateral meniscus: a, proximal surface of medial meniscus; b, proximal surface of lateral meniscus; c, distal surface of medial meniscus; d, distal surface of lateral meniscus; e, cranial extremity of medial meniscus; $f$, caudal extremity of medial meniscus; g, cranial extremity of lateral meniscus; $h$, caudal extremity of lateral meniscus. 1, cranial ligament of medial meniscus; 2, caudal ligament of medial meniscus; 3 , cranial ligament of lateral meniscus; 4, meniscofemoral ligament.
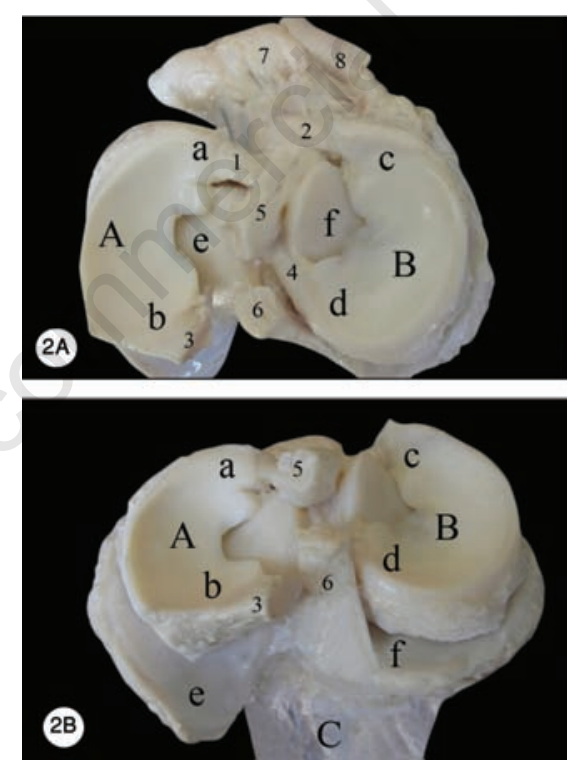

Figure 2. Photograph of left proximal extremity of tibia with menisci of buffalo and their ligamental attachments. A) Lateral meniscus, B) medial meniscus; C) tibia. a, cranial extremity of lateral meniscus; b, caudal extremity of lateral meniscus; c, cranial extremity of medial meniscus; d, caudal extremity of medial meniscus; e, lateral condyle of tibia; $f$, medial condyle of tibia. 1, Cranial ligament of lateral meniscus; 2 , cranial ligament of medial meniscus; 3, meniscofemoral ligament; 4, caudal ligament of medial meniscus; 5 , cranial cruciate ligament; 6 , caudal cruciate ligament; 7 , lateral patellar ligament; 8 , intermediate patellar ligament.
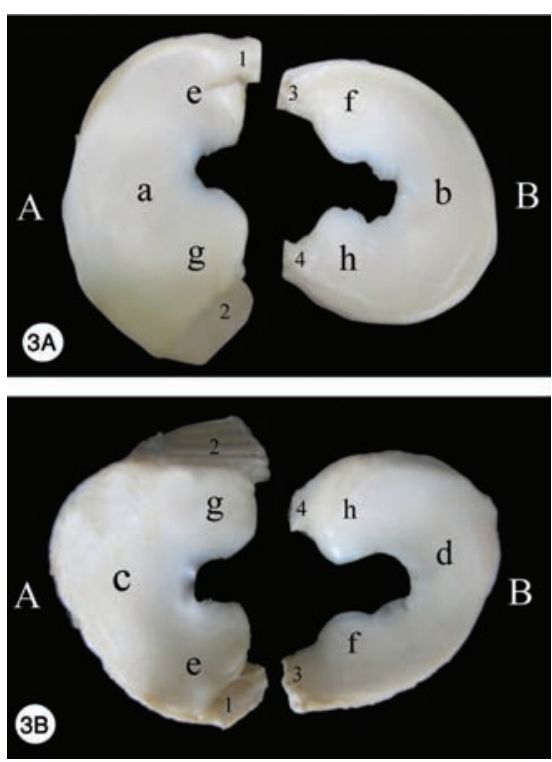

Figure 3. Photograph of menisci of left stifle of cattle. A) Lateral memiscus; B) medial meniscus. a, proximal surface of lateral meniscus; $b$, proximal surface of medial meniscus; c, distal surface of lateral meniscus; $d$, distal surface of medial meniscus; e, cranial extremity of lateral meniscus; $f$, caudal extremity of medial meniscus; g, caudal extremity of lateral meniscus; h, caudal extremity of medial meniscus. 1, cranial ligament of lateral meniscus; 2 , meniscofemoral ligament; 3 , cranial ligament of medial meniscus; 4, caudal ligament of medial meniscus. 
deep for the medial meniscus in buffalo and cattle, respectively, and $12.59 \pm 0.04 \mathrm{~mm}$ and $12.46 \pm 0.02 \mathrm{~mm}$ deep for the lateral meniscus, in buffalo and cattle, respectively.

Sisson and Frandson reported that the menisci in domestic animals help to keep the joint congruent, allow greater range or variety of movement, and also diminish concussion. 6,8 However, Skerritt and McLelland suggested that the main function of the menisci is to provide additional surfaces on which the synovial fluid can exert a lubricating action. ${ }^{9}$

The menisci are peripherally thick and convex, and taper in the center to a thin free margin. The meniscal surfaces conform to the femoral and tibial contours. The medial meniscus is semicircular and the caudal extremity is wider than the cranial extremity. The cranial extremity is attached to the cranial intercondyloid area in front of the cranial cruciate liga-

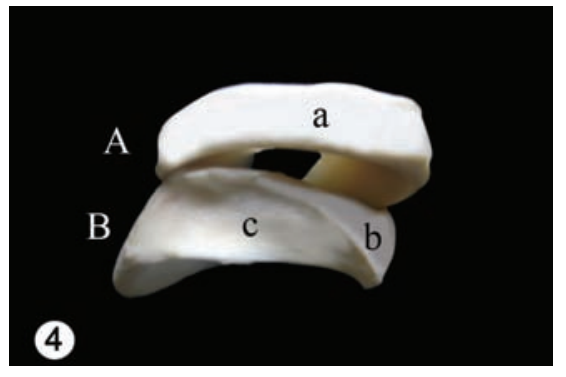

Figure 4. Photograph of menisci of right stifle joint of cattle. Lateral view. A) Medial meniscus; B) lateral meniscus. a, abaxial border of medial meniscus; $b$, abaxial border of lateral meniscus; c, groove on the abaxial border of the lateral meniscus for the popliteus.

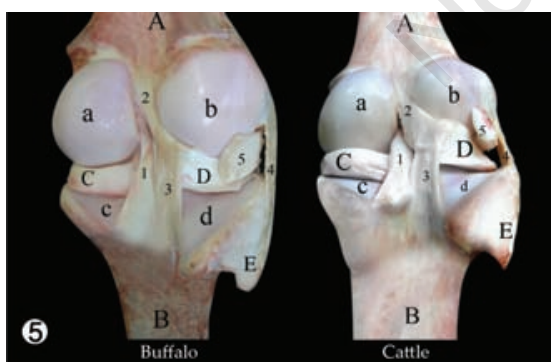

Figure 5. Photograph of right femorotibial joint of buffalo and cattle. Caudal view. A) Femur; B) tibia; C) medial meniscus D) lateral meniscus; E) fibula. a, medial condyle of femur; $b$, lateral condyle of femur; c, medial condyle of tibia; d, lateral condyle of tibia 1 , caudal cruciate ligament; 2, meniscofemoral ligament; 3 , caudal ligament of lateral meniscus; 4, lateral collateral ligament; 5 , tendon of origin of popliteus muscle. ment by the cranial ligament. This attachment is to the cranial surface of tibial condyles.

The caudal extremity of the medial meniscus is firmly attached to the caudal intercondyloid area of the tibia by the caudal ligament of medial meniscus cranial and medial to the caudal cruciate ligament tibial attachment side. Peripherally, the medial meniscus is attached to the medial collateral ligament.

The lateral meniscus is semicircular and covers a larger portion of the tibial condyle than the medial meniscus. The lateral meniscus has cranial and caudal meniscal ligaments anchoring it to the tibia. The cranial ligament of the lateral meniscus attaches its cranial extremity to the cranial intercondyloid area between the two parts of the cranial cruciate ligament. The caudal ligament attaches its caudal extremity to the popliteal notch. This ligament is longer in buffalo $(38.61 \pm 0.14)$ than in cattle $(31.49 \pm 0.19 \mathrm{~mm})$. The lateral

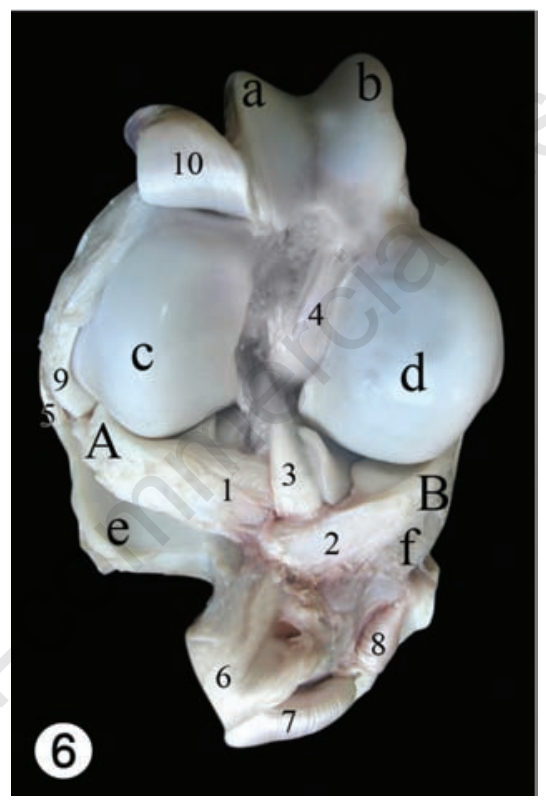

Figure 6. Photograph showing the distal extremity of the femur and proximal extremity of the tibia and their ligamental attachments of right stifle joint of buffalo. The capsules are removed. A) Lateral meniscus; B) medial meniscus. a, lateral ridge of trochlea of femur; $b$, medial ridge of trochlea of femur; c, lateral condyle of femur; $d$, medial condyle of femur; e, lateral condyle of tibia; $f$, medial condyle of tibia. 1, cranial ligament of lateral meniscus; 2 , cranial ligament of medial meniscus; 3) cranial cruciate ligament (twisted); 4, caudal cruciate ligament; 5 , lateral collateral ligament; 6, lateral patellar ligament; 7 , intermediate patellar ligament; 8, medial patellar ligament; 9, tendon of origin of popliteal muscle; 10, common tendon of origin of extensor digitorum longus and Peronaeus tertius mucles.

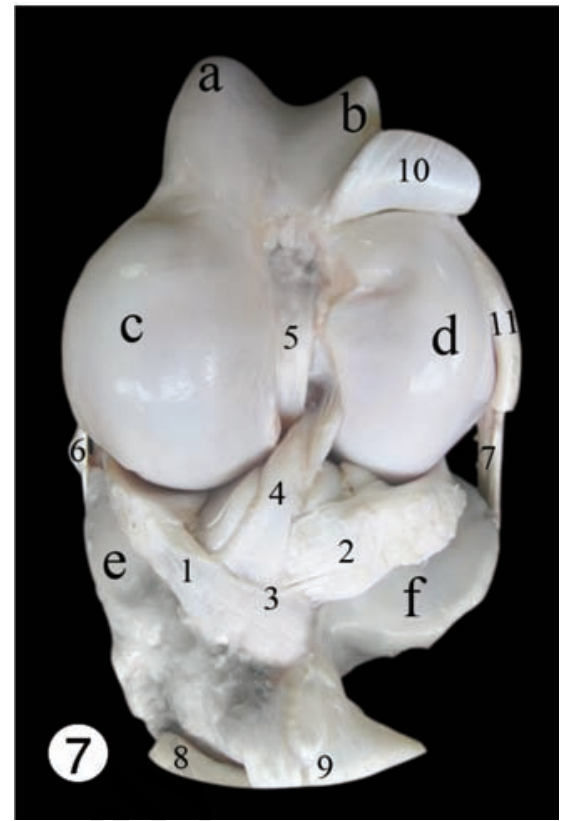

Figure 7. Photograph of the distal extremity of the femur and the proximal extremity of the tibia and their ligamental attachments of left stifle joint of cattle. The capsules are removed. a, medial ridge of trochlea; b, lateral ridge of trochlea; $c$, medial condyle of femur; d, lateral condyle of femur; e, medial condyle of tibia; f, lateral condyle of tibia. 1 , cranial ligament of medial meniscus; 2, cranial ligament of lateral meniscus; 3 , transverse genual ligament; 4, cranial cruciate ligament (Twisted); 5, caudal cruciate ligament; 6 , medial collateral ligament; 7 , lateral collateral ligament; 8, intermediate patellar ligament; 9, lateral patellar ligament; 10, common tendon of origin of long digital extensor and peronaeus tertius muscles; 11, tendon of origin of popliteus muscle.

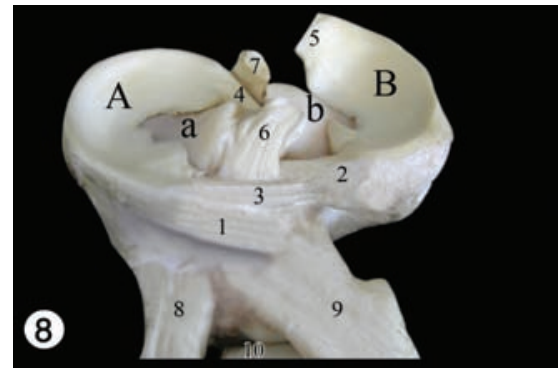

Figure 8. Photograph of proximal extremity of tibia with the menisci and their ligamental attachments of left stifle of cattle. A) medial meniscus; B) lateral meniscus. a, medial condyle of tibia; b, lateral condyle of tibia. 1, cranial ligament of medial meniscus; 2 , cranial ligament of lateral meniscus; 3 , transverse genual ligament; 4 , caudal ligament of medial meniscus; 5 , meniscofemoral ligament; 6 , cranial cruciate ligament; 7 , caudal cruciate ligament; 8, medial patellar ligament; 9, lateral patellar ligament; 10) intermediate patellar ligament. 
meniscus is loose peripherally as it is separated from the lateral collateral ligament by the tendon of origin of the popliteus muscle. In addition, the lateral meniscus has an attachment through the meniscofemoral ligament to the medial condyle of the femur. In cattle, it was observed that a transverse genual ligament connects the cranial extremities of both menisci. When the stifle joint is flexed, the menisci move caudally. Caudal motion of the medial meniscus is guided by its attachment to the medial collateral ligament and is semimembranous, whereas cranial translation is caused by the push of the cranial femoral condyle. 10 The lateral meniscus is stabilized and motion-guided by the popliteus tendon and meniscofemoral ligament.11,12 In bovine, the menisci glide forwards over the tibia as the femoral condyles roll upon them in extension and the restriction of their movement imposed by the meniscal ligaments is an important brake upon straightening the joint.

The present study shows that the dimensions of the meniscal ligaments of buffalo and cattle are nearly the same except the caudal ligament of lateral meniscus and the meniscofemoral ligament. The caudal ligament of lateral meniscus is longer in buffalo than in cattle. Therefore, the femur moves more cranially than normal leading to straightening the joint.

The meniscofemoral ligament is thicker in cattle $(6.55 \pm 0.04 \mathrm{~mm})$ than in buffalo $(3.85 \pm 0.05 \mathrm{~mm})$; therefore, it helps to stabilize the movement of the menisci. Furthermore, the presence of the transverse genual ligament which fixes the two menisci cranially increases the stability and prevents more forward movement of the femur.

From a morphological point of view, the movement between the menisci and the tibia is a gliding movement due to their articular surfaces being nearly flat. As the articular surfaces of the femur and menisci are condylar in shape, this joint is a hinge joint. The menisci are fixed on the tibia by the meniscal ligaments. Therefore, the femur moves on the menisci which are fixed on the tibia.

The stifle joint is a modified hinge joint made up of paired femorotibial and single femoropatellar articulation. 13 The two articulations are interdependent in that the patella is firmly connected to the tibia by ligamentous tissue so that any movement between the femur and the tibia also occurs between the patella and femur (Evans, 1993; data not shown).

The femorotibial joint is the largest joint in the body and is composed of two condyloid articulations. Intervening medial and lateral menisci serve to enhance the conformity of the joint.

Sisson and Frandson et al.6,8 reported that the menisci in domestic animals help to keep the joint congruent, allow greater range or variety of movement, and also diminish concussion. However, Skerritt and McLelland suggested that the main function of the menisci is believed to provide additional surfaces on which the synovial fluid can exert a lubricating action. ${ }^{9}$ When the stifle joint is flexed the menisci move caudally. Caudal motion of the medial meniscus is guided by its attachment to the medial collateral ligament and semimembranouses, whereas cranial translation is caused by the push of the cranial femoral condyle. ${ }^{10}$ The lateral meniscus is stabilized and its movement is guided by the popliteus tendon and meniscofemoral ligament. 11,12

In bovine, the menisci glide forwards over the tibia as the femoral condyles roll upon them in extension and the restriction of their movement imposed by the meniscal ligaments is an important brake upon straightening the joint. 13

\section{Conclusions}

The caudal ligament of lateral meniscus is longer in buffalo than in cattle. Therefore, the femur moves more cranially than normal to straighten the joint. The meniscofemoral ligament is thicker in cattle $(6.55 \pm 0.04 \mathrm{~mm})$ than in buffalo $(3.85 \pm 0.05 \mathrm{~mm})$. Therefore, it helps to stabilize the movement of the menisci. Furthermore, the presence of the transverse genual ligament which fixes the two menisci cranially increases stability and prevents a more forward movement of the femur.

\section{References}

1. Goldblatt JP, Richmond JC. Anatomy and biomechanics of the knee. Oper Techn
Sport Med 2003;11:172-86.

2. Semieka MA, Misk NA. Clinical experience in the diagnosis and surgical treatment of upward fixation of patella in buffaloes. 5th world buffalo congress. Royal Palace Caserta Italy, 1997. pp.648-652.

3. Nomina Anatomica Veterinaria 2005. Fifth Edition. Available from: http//www.wavaamav.org/Downloads/nav_2012.pdf

4. Raghavan D. Anatomy of the ox with comparative notes on the horse, dog and fowl. New Delhi: Indian Council of Agricultural Research; 1964.

5. Morcos BM. The anatomical study of the joints of the limbs of the camel. Degree dissertation. Faculty of Veterinary Medicine, Cairo University, Cairo, Egypt. 1995.

6. Sisson S. Syndesmology. In: Sisson S, Grossman JD, Getty R. Sisson and Grossman's: the Anatomy of the Domestic Animals. Volume 1. London: WB Saunders \& $\mathrm{C} 0 ; 1975$

7. May NDS. The anatomy of the sheep. Brisbane: University of Queensland Press; 1970.

8. Frandson RD, Spurgeon TL. Anatomy and physiology of farm animals. Fifth Edition. Philadelphia: Lea\&Febiger; 1992.

9. Skerrit GC, McLelland J. An introduction to the functional anatomy of the limbs of the domestic animals. Bristol: John Wright \& Sons; 1984.

10. Simon SR, Alaranta H, An KN, et al. Kinesiology. In: Buckwalter JA, Einhorn TA, Simon SR. Orthopaedic basic science. Biology and biomechanics of the musculoskeletal system. 2nd ed. Rosemont: American Academy of Orthopaedic Surgeons; 2000.

11. Heller L, Langman J. The meniscofemoral ligaments of the human knee. J Bone Joint Surg 1964;46:307-13.

12. Staubli HU, Birre S. The popliteus tendon and its fascicles at the popliteal hiatus: gross anatomy and functional arthroscopic evaluation with and without anterior cruciate ligament deficiency. Arthroscopy 1990;6:209-20.

13. Dyce KM, Wensing CJG. Essentials of bovine anatomy. Philadelphia: Lea\& Febiger; 1971. 